Sheridan College

SOURCE: Sheridan Institutional Repository

$1-2007$

\title{
On Damping and Fluidelastic Instability in a Tube Bundle Subjected to Two-Phase Cross-Flow
}

Joaquin Moran

Sheridan College, joaquin.moran@sheridancollege.ca

David S. Weaver

McMaster University

Follow this and additional works at: https://source.sheridancollege.ca/fast_publications

Part of the Mechanical Engineering Commons

\section{SOURCE Citation}

Moran, Joaquin and Weaver, David S., "On Damping and Fluidelastic Instability in a Tube Bundle Subjected to Two-Phase Cross-Flow" (2007). Publications and Scholarship. 61.

https://source.sheridancollege.ca/fast_publications/61

\section{(c) $10(9)$}

This work is licensed under a Creative Commons Attribution-Noncommercial-No Derivative Works 4.0 License. This Conference Proceeding is brought to you for free and open access by the Faculty of Applied Science \& Technology (FAST) at SOURCE: Sheridan Institutional Repository. It has been accepted for inclusion in Publications and Scholarship by an authorized administrator of SOURCE: Sheridan Institutional Repository. For more information, please contact source@sheridancollege.ca. 


\section{PVP2007-26458-DRAFT}

\section{ON DAMPING AND FLUIDELASTIC INSTABILITY IN A TUBE BUNDLE SUBJECTED TO TWO-PHASE CROSS-FLOW}

\author{
Joaquin E. Moran \\ Department of Mechanical Engineering \\ McMaster University \\ Hamilton, Canada L8S 4L7 \\ E-mail: moranje@mcmaster.ca
}

\author{
David S. Weaver \\ Department of Mechanical Engineering \\ McMaster University \\ Hamilton, Canada L8S 4L7 \\ E-mail: weaverds@mcmaster.ca
}

\begin{abstract}
An experimental study was conducted to investigate damping and fluidelastic instability in tube arrays subjected to twophase cross-flow. The purpose of this research was to improve our understanding of these phenomena and how they are affected by void fraction and flow regime. The working fluid used was Freon 11, which better models steam-water than air-water mixtures in terms of vapour-liquid mass ratio as well as permitting phase changes due to pressure fluctuations. The damping measurements were obtained by "plucking" the monitored tube from outside the test section using electromagnets. An exponential function was fitted to the tube decay trace, producing consistent damping measurements and minimizing the effect of frequency shifting due to fluid added mass fluctuations. The void fraction was measured using a gamma densitometer, introducing an improvement over the Homogeneous Equilibrium Model (HEM) in terms of density and velocity predictions. It was found that the Capillary number, when combined with the total damping ratio (interfacial damping), shows a well defined behaviour depending on the flow regime. This observation can be used to develop a better methodology to normalize damping results. The fluidelastic results agree with previously presented data when analyzed using the HEM and the half-power bandwidth method. The interfacial velocity is suggested for fluidelastic studies due to its capability for collapsing the fluidelastic data. The interfacial damping was introduced as a tool to include the effects of flow regime into the stability maps.
\end{abstract}

\section{NOMENCLATURE}

Cap Capillary number

$C_{i} \quad$ Interfacial Coefficient

$D \quad$ Tube diameter $[\mathrm{m}]$

$D_{e} \quad$ Effective tube diameter [m]

$D_{\text {ref }}$ Reference diameter, related to confinement [m]

$f \quad$ Frequency of vibration $[\mathrm{Hz}]$

$G_{p} \quad$ Pitch mass flux $\left[\mathrm{kg} / \mathrm{m}^{2} \mathrm{~s}\right]$

$m$ Lineal mass, including fluid added mass $[\mathrm{kg} / \mathrm{m}]$

$S \quad$ Slip Ratio

$U_{l s} \quad$ Superficial Velocity of the liquid phase $[\mathrm{m} / \mathrm{s}]$

$U_{g s} \quad$ Superficial Velocity of the gas phase $[\mathrm{m} / \mathrm{s}]$

$U_{l} \quad$ Velocity of the liquid phase $[\mathrm{m} / \mathrm{s}]$

$U_{g} \quad$ Velocity of the gas phase $[\mathrm{m} / \mathrm{s}]$

$\delta$ Logarithmic decrement of damping

$\rho_{g} \quad$ Gas density $\left[\mathrm{kg} / \mathrm{m}^{3}\right]$

$\rho_{l} \quad$ Liquid density $\left[\mathrm{kg} / \mathrm{m}^{3}\right]$

$\rho_{t p} \quad$ Two-phase density $\left[\mathrm{kg} / \mathrm{m}^{3}\right]$

$\zeta_{T}$ Total damping ratio

$\zeta_{v} \quad$ Viscous damping component

$\zeta_{s} \quad$ Structural damping component

$\zeta_{t p} \quad$ Two-Phase damping component

$\zeta_{i} \quad$ Interfacial damping

$\zeta_{F} \quad$ Fluidelastic damping

$\sigma$ Surface tension $[\mathrm{N} / \mathrm{m}]$

$\mu_{l} \quad$ Dynamic viscosity of the liquid phase $[\mu \mathrm{Pa} \mathrm{s}]$

$v \quad$ Kinematic viscosity $\left[\mathrm{m}^{2} \mathrm{~s}\right]$ 


\section{INTRODUCTION}

Flow-induced vibrations in heat exchangers are an important concern in the power and process industries, due to their capability for generating tube failures in a relatively short time. This translates into production losses, additional maintenance and, in general, significant financial losses. However, the increasing demand in the performance of these devices requires lighter structures and higher flow rates. These factors make heat exchangers more prone to vibration problems, leading to fretting wear at the tube supports and possibly tube-to-tube clashing. In the nuclear industry, steam generators are particularly sensitive to flow-induced vibration problems, especially at the U-bend of the tube bundle, where two-phase cross-flow occurs. In this cases, avoiding a potential failure is also a matter of safety.

Although the nature of the excitation mechanisms is relatively well understood in single-phase flows (see Paidoussis [1], [2], Weaver and Fitzpatrick [3] and Price [4]), some difficulties arise in two-phase flows, mainly related to the flow characterization.

The mechanisms of excitation in two-phase flows are basically turbulence buffeting and fluidelastic instability (FEI). The latter can produce large oscillations and early failure of the tubes, and has been the main subject of attention from a research point of view. However, the small amplitude vibration produced by the turbulent buffeting should also be considered for long-term design purposes.

From a practical point of view, it has been postulated that the occurrence of FEI can be predicted using the two nondimensional parameters used for single-phase flows: the reduced velocity and the mass-damping parameter. However, in twophase flows, the determination of damping is not straightforward because it is highly dependant on void fraction and flow regime. Therefore, measuring two-phase damping of tube bundles is more difficult than for single-phase flows.

Regarding the characterization of the two-phase flow, previous studies have typically used the Homogeneous Equilibrium Model (HEM) to determine the void fraction and other flow parameters. The HEM assumes that the liquid and gas phases are uniformly distributed and move with the same velocity (no slip). In general, this is not a valid assumption and it certainly does not provide for void segregation and different flow regimes, including intermittent flows. The void fraction is a key parameter when analyzing two-phase flow-induced vibrations because it affects how the flow velocity and two-phase density are determined. Equally important is the nature of the fluids used in the experiments. Most of the early experiments in two-phase flow-induced vibrations were carried out using air-water mixtures, not allowing for the phase change and flashing that can occur in steam-water flows. The reason is due to better control of flow regime and void fraction and lower operating pressures and temperatures. This reduces considerably the cost of the experiments, but at the expense of the physical modelling of the real problem. Refrigerants and other single-component fluids can approximate more accurately the density ratio, surface tension and liquid viscosity of steam-water mixtures.

The purpose of this paper is to present the results of an experimental study designed to improve our understanding of the effects of two-phase flow on damping of tube bundles. A dimensional analysis of the problem is carried out, and a relationship between damping and a dimensionless group is established. This information could lead to better design guidelines to avoid the occurrence of fluidelastic instability.

\section{Damping in Two-Phase Flows}

Carlucci [5] and Carlucci and Brown [6] found that the damping of a cylinder in two-phase air-water flow was much higher than for single-phase flows, either liquid or gas. They also established a relationship between damping and void fraction. In order to take into account the additional damping in two-phase flows, they proposed an expression for the total damping ratio of the form

$$
\zeta_{T}=\zeta_{s}+\zeta_{v}+\zeta_{t p}
$$

where $\zeta_{T}$ represents the total damping ratio, $\zeta_{s}$ is the structural damping of the system, $\zeta_{v}$ is the viscous component due to the presence of the fluid, and $\zeta_{t p}$ is the two-phase contribution (which in turn includes additional flow dependant terms). Pettigrew et al. [7] and Pettigrew and Taylor [8] have used Carlucci's approach to analyze the behaviour of damping of tube bundles in two-phase flows. The structural component can be measured in air, and the viscous damping is determined using an expression proposed by Rogers et al. [9] for single-phase flows

$$
\zeta_{v}=\frac{\pi}{\sqrt{8}}\left(\frac{\rho D^{2}}{m}\right)\left(\frac{2 v}{\pi f D^{2}}\right)^{0.5}\left\{\frac{\left[1+\left(D / D_{e}\right)^{3}\right]}{\left[1-\left(D / D_{e}\right)^{2}\right]^{2}}\right\} .
$$

In equation $2, \rho$ represents the fluid density, $D$ is the tube diameter, $m$ is the mass per unit length (including the fluid added mass), $v$ is the kinematic viscosity, $f$ is the tube frequency and the bracketed term on the right side represents a confinement function. The two-phase component is calculated by subtracting the sum of $\zeta_{s}$ and $\zeta_{v}$ from the total damping ratio $\zeta_{T}$, which is measured using the half-power bandwidth method based on the averaged frequency response of the tube. Even when equation 2 was originally formulated to estimate the "added damping" provided by a single-phase stagnant fluid, Pettigrew et al. [7] assumed that an analogous expression could be used in two-phase flow problems. They replaced the density and viscosity by twophase quantities, with the density defined according to the HEM and the equivalent two-phase viscosity proposed by McAdams (see Pettigrew et al. [7]). Pettigrew and Taylor [8] also presented 
an expression to normalize and compare the damping ratio obtained in two-phase cross-flow experiments.

The relationship between damping and void fraction was further studied by Hara [10], who established that the maximum damping ratio corresponded to a certain void fraction interval (30 to $60 \%$ for his experiments). Axisa et al. [11] presented experiments using steam-water mixtures. They found that damping is indeed related to void fraction, and that the values obtained for steam-water were considerably lower than those for air-water mixtures, at least for void fractions over $80 \%$ (based on the Homogeneous Equilibrium Model). This is an indicator of the significant differences which may be found between experiments carried out with air-water and those using one-component mixtures.

Nakamura et al. [12] carried out experiments in in-line arrays to measure two-phase damping and added mass in twophase flows. They used steam-water mixtures at high pressure (up to $5.8 \mathrm{MPa}$ ), and implemented an electromagnetic device to excite a metal block mounted on the tube support wires. They found that the damping ratio changes depending on the location of the tube within the array, and the inclination of the bundle respect to the direction of the flow is also a factor to be considered. They also found that damping was larger in the lift direction than in the drag direction. In order to analyze and compare their results, they used the two-phase damping formulation proposed by Axisa et al. [11].

The way that damping is estimated from experimental data is also an important issue. Moran and Weaver [13] proposed an alternative damping measurement technique that can be used in experiments with two-phase flow across a tube array, which is confined in a test section. The device can produce a steady and controlled oscillation of the tube in a non-intrusive fashion. A pair of electromagnetic coils was used to achieve this purpose. Once the electromagnets are shut off, the decay trace of the tube can be captured using strain gauges. This allowed a comparison between the traditional half-power bandwidth method against the logarithmic decrement and the curve fitting approaches for tube bundle vibrations in two-phase flow. It was found that the halfpower bandwidth method overpredicts the damping when compared to the exponential fitting to the decay curve, more noticeably in bubbly and intermittent flows.

The normalization procedure most commonly used for tube bundle damping analysis was introduced by Pettigrew et al. [7], in order to be able to compare results from different researchers. The objective was to develop design guidelines from experimental results obtained using different fluids and tube array configurations. The normalization was applied only to the two-phase component of the total damping ratio.

$$
\left(\zeta_{t p}\right)_{D}=\zeta_{t p}\left(\frac{m}{\rho_{l} D^{2}}\right)\left\{\frac{\left[1-\left(D / D_{e}\right)^{2}\right]^{2}}{\left[1+\left(D / D_{e}\right)^{3}\right]}\right\}
$$

where $\rho_{l}$ is the density of the liquid phase. In equation 3 , two terms are used for the normalization: the first is the mass ratio, based on the density on the liquid and accounts for the added mass effects; the second, based on the tube diameter, represents the contribution of tube confinement. More recently, in an attempt to find a relationship between damping and other flowrelated parameters, Pettigrew and Knowles [14] reported results for a single cylinder subjected to two-phase axial flow. They concluded that the effect of surface tension on two-phase damping was strong, and tried to include it into the normalized form of the two-phase damping. However, the relationship between damping and surface tension was complex, and different for each flow regime. A surface tension term could not be included in the formulation.

Baj and de Langre [15] used a different approach. They considered the total damping ratio to be comprised of only two components, structural and fluid, the latter including all the effects related to the two-phase mixture flow. In order to improve the stagnant-flow defined damping, a new normalization procedure was introduced, in which the liquid density was replaced by the two-phase density. The resultant normalized fluid damping ratio is

$$
\zeta_{n}^{f}=\zeta^{f}\left(\frac{m}{\rho_{t p} D^{2}}\right)\left(\gamma \frac{D}{D_{r e f}}\right)
$$

In equation $4, \zeta_{n}^{f}$ is the normalized fluid damping, $\rho_{t p}$ is the density of the mixture and the terms $D_{\text {ref }} / D$ and $\gamma$ take into account the confinement effects. This equation assumes that the fluid damping is linearly dependent on the tube diameter, as in single-phase flow. They argued that damping in two-phase flow should be treated as a single term rather than separate terms, as proposed by Pettigrew et al. [7]. By extrapolating the values of the normalized fluid damping to zero reduced velocity, Baj and de Langre introduced the concept of "quiescent fluid damping" in two-phase mixtures. The study showed that the quiescent fluid damping was lower than that recommended by the current design guidelines (which provide the damping measured at half the mass flux required for fluidelastic instability and assume that damping is independent of mass flux). In addition, the authors introduced a new "two-phase vibrational viscosity", arguing that the additional damping observed in two-phase flow is due to purely viscous effects. The new viscosity also proved to be much higher than the predictions of the existing models for two-phase mixtures (McAdams, Duckler and Cicchitti, see Collier [16]).

\section{Fluidelastic Instability in Two-Phase Flows}

As mentioned above, it has been assumed that the massdamping parameter and the reduced velocity can predict the fluidelastic instability in two-phase flows. However, the velocity and density must be defined for the two-phase mixture in order 
to determine those parameters. Pettigrew et al. [7] suggested to use the pitch velocity based on the HEM density for fluidelastic analysis. Again, the overestimation of density caused by the HEM may affect the reduced velocity and compromise the design. Other models have been proposed to determine a representative velocity of the two-phase mixture, including the equivalent velocity introduced by Feenstra et al. [17] and the interfacial velocity, used by Nakamura et al. [18]. The latter represents a very interesting approach, because it can be used for any flow regime and takes into account the density ratio effects. From a fluidelastic point of view, the use of the interfacial velocity notably reduces the scatter in the data. In this study, the pitch velocity was used to compare the present results to previous research. Then, the interfacial velocity was implemented to gain physical insight into the fluidelastic phenomena in two-phase flows.

\section{EXPERIMENTAL FACILITY Two-Phase Flow Loop}

The experiments were carried out in a two-phase flow loop located at McMaster University. The working fluid is Refrigerant 11 (Freon), and is evaporated using a set of heaters located below the test section (see Figure 1). The heaters are capable of transmitting up to $48 \mathrm{~kW}$ of power to the fluid. A gear pump is used to circulate the Freon throughout the loop. The maximum pitch mass flux attained by the pump (based on single-phase flow) is $1000 \mathrm{~kg} / \mathrm{m}^{2} \mathrm{~s}$. A condenser located downstream the test section removes the heat from the mixture and allows for a better control of the thermodynamic parameters in the test section. A detailed description of the flow loop can be found in Feenstra et al. $[17,19]$.

\section{Test Section and Model Tube Array}

The test section where the model bundle is located, has a rectangular cross-section of $49.2 \mathrm{~mm}$ by $197 \mathrm{~mm}$. It has two glass windows on the sides for observation, as well as a frontal window. The test section has half-tubes attached to the sides, in order to minimize the effect of the flat walls on the flow configuration.

The array consists of ten cantilever-mounted brass tubes, with an external diameter of $9.525 \mathrm{~mm}$ (0.375 in). The geometric pattern of the bundle is a parallel triangle, with a pitch-overdiameter ratio of 1.49 , similar to that in CANDU steam generators. The tubes were tuned to within $\pm 1 \%$ of the average natural frequency measured in air. For the monitored tube, the natural frequencies in liquid and vapour Freon were $41.75 \mathrm{~Hz}$ and 48.25 $\mathrm{Hz}$ respectively.

The vibratory response of the tube was measured by using two strain gauges, located on the cylindrical support between the tubes and the base plate of the array (see Figure 2). These strain gauges were positioned at 90 degrees from each other, allow-
Figure 1. Diagram of the two-phase flow loop and tube array geometry: (1) Main pump, (2) orifice plates for mass flux measurement, (3) flow control valves, (4) heaters, (5) test section, (6) shell-and-tube condenser, (7) gamma densitometer.

ing for the measurement of displacement in both the transverse and stream-wise directions. For the damping measurements, the monitored tube is excited to vibrate only in the lift direction. Hence, the damping reported in this study corresponds only to the transverse plane. The output signal was collected using a data acquisition card and a dynamic analyzer (HP 35670A). The data acquisition card provided the time history information of the monitored tube, while the dynamic analyzer provided the averaged frequency response. For each trial, the dynamic analyzer collected a total of 100 averages from 0 to $100 \mathrm{~Hz}$, with a resolution of $0.25 \mathrm{~Hz}$.

\section{Electromagnetic Excitation Device}

Figure 2 shows a diagram of the electromagnetic coils in position. The end-cap of the monitored tube has a permanent magnet attached, which improves the response of the tube to the electromagnetic field imposed. The polarity of these temporal magnets is changed at a frequency equal to the resonant frequency of the tube, producing the excitation required to obtain the decay trace. Since the excitation comes from outside the test section, the method is non-intrusive, that is, it does not interfere with the two-phase flow distribution across the bundle or around the monitored tube. 
Figure 2. Side view of the test section: Mounted tube, strain gauges and electromagnetic device.

\section{Experimental Procedure}

For each experiment, the pitch mass flux was held constant while the void fraction was changed by increasing the heat transferred to the fluid. The pitch mass fluxes studied ranged from 100 to $500 \mathrm{~kg} / \mathrm{m}^{2} \mathrm{~s}$ in steps of $50 \mathrm{~kg} / \mathrm{m}^{2} \mathrm{~s}$. The temperature of the Freon was measured at several points along the loop, including locations upstream and downstream of the test section, at the heaters and downstream of the condenser. When the temperatures had remained constant for a certain period of time, then the void fraction and tube response were recorded. Waiting for "steady state" ensures that the flow regime and void fraction will not change while the measurements are performed. The averaged frequency spectra of the tube were captured while the void fraction was measured directly using the gamma densitometer. The latter was located upstream of the model tube bundle, as shown in Figure 1.

Two series of experiments were carried out. For the first (Series A), only the monitored tube was flexible, while all the other tubes were fixed. This feature allowed measurement of the damping caused by the two-phase flow on the monitored tube. In order to obtain the decay trace of the tube, the electromagnets were turned on after the frequency spectra and the void fraction had been recorded. They remained working for a period of 5 to 10 seconds to ensure that the tube was vibrating at a constant amplitude. Then, the power was shut-off, and the tube decay response was captured by the strain gauges. This procedure was repeated three times for each value of heat flux, and then averaged to obtain a representative damping ratio. The second series (Series B) was performed with a fully-flexible array, which permitted us to determine the critical velocity for the tube bundle for each mass flux.
Figure 3. Exponential Fitting to the decay trace. At point $a$, the electromagnets are shut-off. The interval between $b$ and $c$ is used for the fitting, avoiding the turbulent excitation seen at point $d$.

\section{DAMPING MEASUREMENT METHOD}

In this study, the damping was determined by fitting an exponential function to the decay trace (see Figure 3). For each flow velocity, three damping measurements were obtained and averaged. This procedure produces a more consistent and reliable estimate of damping, especially when the void fraction fluctuations produce intense frequency shifting [13]. Figure 4 shows how the half-power bandwidth method overpredicts the damping ratio for void fractions below $50 \%$, mostly coinciding with the bubbly flow regime. The effect of frequency shifting diminishes as the flow pattern changes to intermittent and dispersed. After the decay response is recorded, a program in MATLAB performs a least squares fitting using the peaks maxima. The function used was

$$
y=A e^{-B t}, \text { where } B=\zeta \omega_{n} .
$$

\section{Two-Phase Flow Modelling}

In two-phase flows, experience has shown that damping is strongly dependent on void fraction and flow regime. As a result, a reliable measurement of void fraction is a key element in the analysis of two-phase damping and fluidelastic instability. Traditionally, the Homogeneous Equilibrium Model (HEM) has been used for the estimation of void, because its ease of application. However, the HEM assumes that there is no slip between the liquid and gas phases, that is, they flow together at the same velocity. For the case of vertical-upwards gas-liquid flows, the 
obtaining the following groups:

$$
\begin{aligned}
& \Pi_{1}=W e / \operatorname{Re}=\text { Cap }=\mu_{l} U_{g s} / \sigma \\
& \Pi_{2}=P / D \\
& \Pi_{3}=F r=V_{p}^{2} / D g \\
& \Pi_{4}=\Delta \rho / \rho_{t p} \\
& \Pi_{5}=\alpha \\
& \Pi_{6}=S .
\end{aligned}
$$

Figure 4. Comparison between the half-power bandwidth method and the exponential fitting method for a mass flux of $250 \mathrm{~kg} / \mathrm{m}^{2} \mathrm{~s}$ [13].

buoyancy contributes to accelerate the gas phase, causing a velocity difference that should not be neglected.

As an alternative, Feenstra et al. [17] used gamma densitometry in order to improve the two-phase density and velocity calculations. This is especially important when the flow regime changes from bubbly to intermittent, because the unsteadiness and turbulence present in the flow departs from the physical behaviour assumed in the HEM. In this study, the void fraction (RAD Void - Radiation Attenuation Determination) was measured using a gamma densitometer, but also the HEM void was calculated for comparison purposes.

\section{EXPERIMENTAL RESULTS Dimensional Analysis of Damping}

With the objective of investigating the relationship between the damping present in the system and the two-phase flow parameters, a dimensional analysis was carried out for the damping ratio. The idea was not to find an expression to evaluate the damping, but to find a relationship between the latter and the properties of the two-phase flow. As a first step, the total damping ratio in a tube bundle was assumed to be a function of the following flow variables:

$$
\zeta=\zeta\left(\alpha, S, G_{p}, \mu_{l}, \rho_{t p}, \Delta \rho, P, D, \sigma, g\right)
$$

where $S$ is the slip ratio $\left(U_{g} / U_{l}\right), G_{p}$ is the mass flux, $\sigma$ is the surface tension, $\Delta \rho$ is the density difference between the liquid and gas phases, $P$ is the array pitch, $D$ is the tube diameter and $g$ is the gravitational acceleration. The resulting dimensionless parameters were combined and rearranged to reduce its number,
In equation 7, Cap is the Capillary number, the ratio of the Weber number to the Reynolds number. The mass flux $G_{p}$ has been substituted by the superficial velocity of the gas phase. The other parameters are the Froude number $(F r)$, the density ratio $\Delta \rho$, the pitch ratio and the slip ratio, which were originally dimensionless. The Capillary number has a special significance in two-phase flow across tube bundles. It was first introduced by Feenstra et al. [20] in order to calculate the slip ratio and determine void fraction. In Feenstra's void fraction model, the Capillary number represents the ratio of viscous forces to surface tension (interfacial) forces, which affect the development and transition between flow regimes. We know that surface tension has a strong influence on two-phase damping [14] and, as the liquid phase carries much of the flow momentum, the liquid viscosity was included instead of using a two-phase approach. Since Feenstra's void fraction model has proven to be very effective in reproducing the behaviour of two-phase mixtures (see Consolini et al. [21]), both for single-component (steam-water mixture, refrigerants) and two-component flows (air- water), it is very probable that the use of the Capillary number will allow us to capture some of the physics involved. In this study, the viscosity of the liquid phase and the surface tension were determined based on the temperature of the mixture, measured just upstream of the tube bundle. The superficial velocity of the gas was calculated as

$$
U_{g s}=\frac{x G_{p}}{\rho_{g}}
$$

where $x$ is the thermodynamic quality and $\rho_{g}$ is the density of the gas phase. The total damping ratio can be combined with the Capillary number as a normalization parameter. Figure 5 shows the combination of the total damping ratio and the Capillary number, called Interfacial Damping Parameter $\left(\zeta_{i}\right)$, versus the void fraction. The flow regimes were determined based on the flow regime map proposed by Ulbrich and Mewes [22] and corroborated by visual observation. All the mass fluxes studied are shown. For the first time is it shown clearly how flow regime affects the damping. For the bubbly flow regime, we can 
Figure 5. Interfacial damping parameter as a function of void fraction, after the LOESS procedure has been applied. Note that the interfacial damping parameter remains roughly constant during the intermittent flow regime.

observe a remarkable collapse of the damping data, even when recent experiments have shown that damping is dependent on mass flux for a certain range of void fractions (see Moran and Weaver [13]). After the two-phase flow becomes intermittent, the trend becomes less apparent, while for dispersed flows the collapse is again good. The large scatter observed during the intermittent flow regime is probably due to the random and large spatial and temporal changes in local void fraction. On the other hand, the good agreement observed in the bubbly and dispersed flow regimes may indicate that the damping mechanism is similar to that in single-phase flows. With the objective of extracting more information from this particular plot, an statistical procedure known as LOESS (locally weighted polynomial regression) was implemented. The LOESS procedure is useful when we need to find trends in large clouds of data points (see Cleveland [23]). The result is also observed in Figure 5. As mentioned above, the trend is clear for bubbly and dispersed flows. During the intermittent flow regime, the interfacial damping parameter seems to remain constant. Two combined mechanisms are taking place here: the total damping ratio is decreasing due to the proximity of the critical velocity and the reduction of the added mass, and the superficial velocity of the gas phase is increasing, due to the increase in void fraction. These effects tend to level each other during the intermittent flow regime, where the Capillary number increases exponentially with the void fraction. After the dispersed flow regime started, even the continuous increase of the inertial effects could not counteract the diminishing of the damping present in the system, due to a drop in flow density and the presence of fluidelastic forces.
Figure 6. Stability map showing the fluidelastic data. The reduced velocity and the mass damping parameter are based on HEM density. The half-power bandwidth method was used for the damping.

\section{Fluidelastic Instability}

Figure 6 shows the results obtained for fluidelastic instability, as well as from previous researchers. In this Figure, the pitch velocity is based on the HEM density and the damping reported is calculated with the half-power bandwidth method. The objective is to compare and validate the fluidelastic results obtained in the present research. The results agree with the existing data, and follow the trend indicated by Connors equation (for $K=3$ ). The reduced velocity can also be calculated based on the interfacial velocity introduced by Nakamura et al. [18], defined as

$$
V_{i}=C_{i}\left(U_{g s}+U_{l s}\right)+\sqrt{g D\left(\rho_{l}-\rho_{g}\right) / \rho_{l}},
$$

where $U_{g s}$ and $U_{l s}$ are the superficial velocities of the gas and liquid phases respectively, $g$ is the gravitational acceleration and $C_{i}$ is a coefficient that depends on the geometric configuration of the tube array. For the case of parallel triangular arrays, $C_{i}=0.77$. Figure 7 shows the same results if the interfacial velocity and the RAD density are used in the stability map. The agreement between the different data sets improves dramatically. Note that the use of the RAD density shifts the data to the left when compared to Figure 6. The reason is that the Homogeneous model overpredicts the density of the mixture due to its assumption of no-slip between the phases.

\section{Interfacial Damping and Fluidelastic Instability}

After examining the results shown in Figure 5, an effort was made to incorporate the trend observed into the fluidelastic analysis. This is based on the idea that the mechanism of two-phase 
Figure 7. Stability map showing the fluidelastic data. The reduced velocity is based on the interfacial velocity, and the mass damping parameter is calculated using the RAD density.

damping is different for each flow regime, and as a consequence, they should be taken into account differently for fluidelastic analysis purposes. In order to do this, the damping for fluidelastic analysis, or fluidelastic damping $\left(\zeta_{F}\right)$, was determined by fitting a function to the smoothed line obtained from the LOESS. The fitting is different for each flow regime. For bubbly flow, a quadratic function is used, while the intermittent and dispersed flow regimes are approximated using a straight line. The fluidelastic damping is then determined by

$$
\zeta_{i}=f(\alpha)=\zeta \operatorname{Cap} \Rightarrow \zeta=\zeta_{F}=\frac{f(\alpha)}{C a p}
$$

where $f(\alpha)$ are functions of the void fraction that depend on flow regime and Cap is based on the critical superficial gas velocity. The functions obtained were

$$
\begin{array}{ll}
f(\alpha)=0.16 \alpha^{2}+0.015 \alpha \quad & \text { (Bubbly) } \\
f(\alpha)=0.045 & \text { (Intermittent) } \\
f(\alpha)=-0.18 \alpha+0.18 \quad \text { (Dispersed) }
\end{array}
$$

If the interfacial damping is used to compute the massdamping parameter based on flow regime, we obtain the stability map shown in Figure 8. The trends are clearly different for each flow regime. For bubbly flow, the data follows the Connors' predictions, indicating that the mechanism of damping is probably similar to that in single-phase flows. Once the intermittent flow regime is reached, the critical reduced velocity starts dropping as the critical void fraction increases. This trend has been observed
Figure 8. Stability map based on interfacial damping. Each flow regime has a well defined behaviour. For the case of dispersed flow, the massdamping parameter shows unexpected results.

in the past by Feenstra et al. [17]. The data points corresponding to the dispersed flow regime show an unexpected result. As the critical void fraction increases, the mass-damping parameter actually decreases. On one hand, this is logical from the damping point of view because damping decreases drastically as the vapour phase is approached. However, the changes in the mixture density should overcome this effect. In the limiting case, when the damping in air and the density of Freon vapour are used, the mass-damping parameter is 2.41. More research and analysis is required to better understand the relation between the damping mechanisms for each flow regime, and how this relation can be used for improving the design guidelines to predict the occurrence of fluidelastic instability.

\section{CONCLUSIONS}

Flow-induced vibration experiments were conducted with the objective of studying two-phase damping and its relationship to the flow parameters. Two sets of experiments were carried out, in order to both measure the damping and determine the critical velocity for a parallel triangular tube array. A comparison was made with other researchers data, showing that when the traditional HEM density and the half-power bandwidth damping were used, a good agreement between those studies and the present investigation was observed. A summary of findings of this study are as follows:

1. Even though the characterization of the flow conditions is a difficult task in two-phase flows, the Capillary number seems to capture part of the flow dynamics. From this study, it is clear that damping is a function of flow regime. 
2. The interfacial damping, which is a function of the Capillary number, collapses the data for bubbly and dispersed flows, and its highly variable during the intermittent flow regime. The reason for the collapse is probably the similitude between the damping mechanism in single-phase flows and that in bubbly and dispersed flows. The intermittent flow regime is characterized by a random distribution of void fraction, both temporal and spatial, generating the scatter in the data.

3. If the interfacial damping is used to calculate the massdamping parameter, the trend observed suggests that it produces problems in predicting the fluidelastic instability. This result questions the use of damping in two-phase flow fluidelastic analysis. More research is required to gain insight into how two-phase energy dissipation is taken into account for fluidelastic instability purposes.

\section{REFERENCES}

[1] Païdoussis, M. P., 1982. "A Review of Flow-Induced Vibrations in Reactors and Reactor Components". Nuclear Engineering and Design, 74, pp. 31-60.

[2] Païdoussis, M. P., 2006. "Real-life experiences with flowinduced vibration". Journal of Fluids and Structures, 22, pp. 741-755.

[3] Weaver, D. S., and Fitzpatrick, J. A., 1988. "A review of cross-flow induced vibrations in heat exchanger tube arrays". Journal of Fluids and Structures, 2, pp. 73-93.

[4] Price, S. J., 1995. "A Review of the Theoretical Models for Fluidelastic Instability of Cylinder Arrays in Cross-Flow". pp. 463-518.

[5] Carlucci, L. N., 1980. "Damping and hydrodynamic mass of a cylinder in simulated two-phase flow". Journal of Mechanical Design, 102, pp. 597-602.

[6] Carlucci, L. N., and Brown, J. D., 1983. "Experimental studies of damping and hydrodynamic mass of a cylinder in confined two-phase flow". Journal of Vibration, Acoustics, Stress and Reliability in Design, 105, pp. 83-89.

[7] Pettigrew, M. J., Taylor, C. E., and Kim, B. S., 1989. "Vibration of tube bundles subjected to two-phase cross-flow: Part I - hydrodynamic mass and damping". Journal of Pressure Vessel Technology, 111, pp. 466-477.

[8] Pettigrew, M. J., and Taylor, C. E., 1994. "Two-phase flowinduced vibration: An overview". Journal of Pressure Vessel Technology, 116(3), pp. 233-253.

[9] Rogers, R. G., Taylor, C. E., and Pettigrew, M. J., 1984. "Fluid effects on multispan heat exchanger tube vibration". In ASME PVP Conference.

[10] Hara, F., 1987. "Vibration of circular cylindrical structures subjected to two-phase flows". JSME International Journal, 30(263), pp. 711-722.

[11] Axisa, F., Wullschleger, M., and Villard, B., 1988. "Two- phase cross-flow damping in tube arrays". In ASME PVP Conference, Vol. 133.

[12] Nakamura, T., Hirota, K., Wanatabe, Y., Mureithi, N. W., Kusanabe, T., and Takamatsu, H., 2002. "Dynamics of an in-line tube array subjected to Steam-Water Cross-Flow. Part I: Two-phase damping and added mass". Journal of Fluids and Structures, 16(2), pp. 123-136.

[13] Moran, J. E., and Weaver, D. S., 2006. "Damping measurements in tube bundles subjected to two-phase crossflow". In ASME Pressure Vessels and Piping Conference (PVP2006).

[14] Pettigrew, M. J., and Knowles, G. D., 1997. "Some aspects of heat exchanger tube damping in two-phase mixtures". Journal of Fluids and Structures, 11, pp. 929-945.

[15] Baj, F., and de Langre, E., 2003. "Scaling of damping induced by bubbly flow across tubes". Journal of Fluids and Structures, 17, pp. 351-364.

[16] Collier, J. G., and Thome, J. R., 1996. Convective Boiling and Condensation, 3 ed. Clarendon Press.

[17] Feenstra, P. A., Weaver, D. S., and Judd, R. L., 2002. "Modelling two-phase excited damping and fluidelastic instability in tube arrays". Journal of Fluids and Structures, 16(6), pp. 811-840.

[18] Nakamura, T., Hirota, K., and Tomomatsu, K., 2000. "Some problems on the estimation of flow-induced vibration of a tube array subjected to two-phase flow". In FlowInduced Vibrations, S. Ziada and T. Staubli, eds., A. A. Balkema.

[19] Feenstra, P. A., Weaver, D. S., and Nakamura, T., 2003. "Vortex shedding and fluidelastic instability in a normal square tube array by two-phase cross-flow". Journal of Fluids and Structures, 17, pp. 793-811.

[20] Feenstra, P. A., Weaver, D. S., and Judd, R. L., 2000. “An improved void fraction model for two-phase cross-flow in horizontal tube bundles". International Journal of Multiphase Flow, 26, pp. 1851-1873.

[21] Consolini, L., Robinson, D., and Thome, J. R., 2006. "Void fraction and two-phase pressure drops for evaporating flow over horizontal tube bundles". Heat Transfer Engineering, 27(3), pp. 5-21.

[22] Ulbrich, R., and Mewes, D., 1994. "Vertical, upward gasliquid two-phase flow across a tube bundle". International Journal of Multiphase Flow, 20(2), pp. 249-272.

[23] Cleveland, W. S., 1994. The elements of graphing data, revised ed. Hobart Press. 